\title{
Cortical Pathways or Mechanism in the Face Inversion Effect in Patients with First-Episode Schizophrenia
}

\author{
Yi Liu' \\ Taiyong $\mathrm{Bi}{(\mathbb{D})^{2}}^{2}$ \\ Qijie Kuang' \\ Bei Zhang ${ }^{3}$ \\ Huawang $\mathrm{Wu}^{\prime}$ \\ Haijing $\mathrm{Li}^{\prime}$ \\ Bin Zhang (D' \\ Jingping Zhao ${ }^{4}$ \\ Yuping Ning' \\ Shenglin She' \\ Yingjun Zheng' \\ 'Department of Psychiatry, The Affiliated \\ Brain Hospital of Guangzhou Medical \\ University (Guangzhou Huiai Hospital), \\ Guangzhou, Guangdong, 510370 , People's \\ Republic of China; ${ }^{2}$ Centre for Mental \\ Health Research in School of \\ Management, Zunyi Medical University, \\ Zunyi, Guizhou, 563000, People's \\ Republic of China; ${ }^{3}$ General and \\ Experimental Psychology, Department of \\ Psychology, LMU, Munich, Germany; \\ ${ }^{4}$ Mental Health Institute of the Second \\ Xiangya Hospital, Central South \\ University; Chinese National Clinical \\ Research Center on Mental Disorders; \\ Chinese National Technology Institute on \\ Mental Disorders; Hunan Key Laboratory \\ of Psychiatry and Mental Health, \\ Changsha, Hunan, 4I00II, People's \\ Republic of China
}

Correspondence: Yingjun Zheng; Shenglin

She

Email brainzheng@gzhmu.edu.cn;

shenglinshe@gzhmu.edu.cn
Objective: Impaired face perception is considered as a hallmark of social disability in schizophrenia. It is widely believed that inverted faces and upright faces are processed by distinct mechanisms. Previous studies have identified that individuals with schizophrenia display poorer face processing than controls. However, the mechanisms underlying the face inversion effect (FIE) in patients with first-episode schizophrenia (FSZ) remain unclear.

Methods: We designed an fMRI task to investigate the FIE mechanism in patients with schizophrenia. Thirty-four patients with FSZ and thirty-five healthy controls (CON) underwent task-related fMRI scanning, clinical assessment, anhedonia experience examination, and social function and cognitive function evaluation.

Results: The patients with FSZ exhibited distinct functional activity regarding upright and inverted face processing within the cortical face and non-face network. These results suggest that the differences in quantitative processing might mediate the FIE in schizophrenia. Compared with controls, affected patients showed impairments in processing both upright and inverted faces; and for these patients with FSZ, upright face processing was associated with more severe and broader impairment than inverted face processing. Reduced response in the left middle occipital gyrus for upright face processing was related to poorer performance of social function outcomes evaluated using the Personal and Social Performance Scale.

Conclusion: Our data suggested that patients with FSZ exhibited similar performance in processing inverted faces and upright faces, but were less efficient than controls; and for these patients, inverted faces are processed less efficiently than upright faces. We also provided a clue that the mechanism under abnormal FIE might be related to an aberrant activation of non-face-selective areas instead of abnormal activation of face-specific areas in patients with schizophrenia. Finally, our study indicated that the neural pathway for upright recognition might be relevant in determining the functional outcomes of this devastating disorder.

Keywords: first-episode schizophrenia, face inversion effect, task-related fMRI, face recognition, social functional outcome

\section{Introduction}

Successful social interactions require efficient decoding of information from facial expressions. Not surprisingly, impaired face perception is considered a hallmark of social disability in schizophrenia. Patients with schizophrenia present with psychotic symptoms, emotional and cognitive impairments, and abnormal social communication and interactions. Thus, dysfunction in face processing in schizophrenia seems to be correlated to social functioning impairment. 
An increasing number of studies have identified that individuals with schizophrenia display poorer face processing performance than controls. For instance, according to studies using behavioral face processing tasks, self-face recognition is impaired in patients with schizophrenia. ${ }^{1}$ These studies on self-face recognition have shown global impairment in famous faces recognition and self-face recognition, ${ }^{2,3}$ associated with the theory of mind. ${ }^{4}$ Eyetracking research revealed that patients with schizophrenia present a restricted visual scanning strategy featured by fewer and longer fixations, reduced saccades, less attention to striking facial characteristics in the passive viewing, and avoidance of relevant face features. ${ }^{5,6}$ However, the scan paths of patients with schizophrenia did not differ from that of controls when asked to focus on and determine the features of the faces. ${ }^{6}$

Most of previous studies have shown a dysfunction in the processing of upright faces in patients with schizophrenia; $;^{7-10}$ however, little work has been done on the processing of inverted faces in those with this disorder. ${ }^{11}$ Previous neuropsychological and developmental evidence suggests that the processing of inverted faces is qualitatively distinct from that of upright faces. For instance, prosopagnosia patients with a selective impairment of face perception paradoxically performed better at recognizing inverted faces than upright faces. ${ }^{12}$ Patients with object agnosia and dyslexia caused by closed-head injuries showed normal recognition of upright faces, even though their processing of inverted faces was severely impaired. ${ }^{13,14}$ These findings suggest that the recognition of inverted faces may engage mechanisms distinct from that of upright faces.

There is consistent evidence that face recognition involves a broadly distributed and interconnected brain networks, which include the fusiform face area (FFA) ${ }^{10}$ located in the lateral fusiform gyrus and superior temporal sulcus, ${ }^{10,15}$ as well as the occipital face area (OFA) ${ }^{10,16}$ located in the inferior occipital gyrus, inferior frontal gyrus, ${ }^{17,18}$ and the amygdala. Structural magnetic resonance imaging (sMRI) studies on FFA have found bilaterally reduced volume of gray matter in the fusiform gyrus in patients with schizophrenia, ${ }^{7,19}$ and this volume reduction was significantly correlated with poor performance on delayed facial memory. ${ }^{7}$ However, functional studies achieved mixed results regarding FFA activation, some showing greater activity in the fusiform gyrus when patients performed a gender discrimination task, ${ }^{20}$ others exhibiting lower blood oxygen level dependent responses, longer reaction times, and less accuracy during recognition of famous or unfamiliar faces. ${ }^{21}$ Other functional studies found no activation of the fusiform gyrus in the facial discrimination task, ${ }^{22}$ or showed sporadic activations when patients passively viewed face images.

The face-inversion effect (FIE) typically indicates more difficulty in recognizing inverted faces than recognizing upright faces. FFA is the most consistently observed brain locus involved in the FIE. However, the role of FFA in FIE is yet to be elucidated. One study found that only FFA positively correlated with the behavioral FIE and presented greater neural sensitivity to upright faces than inverted faces across healthy subjects. ${ }^{23}$ Other studies found no change in the magnitude of response for inverted vs upright faces in the FFA. ${ }^{24}$ The inconsistency of these imaging and behavioral data suggests that FIE may also be mediated by other face-selective areas, or even by nonface-selective areas. ${ }^{25}$ The study of Epstein et al also revealed a greater response in lateral occipital object area and the right middle fusiform object area for recognition of inverted faces vs upright faces. ${ }^{24}$

Recent behavioral and computational evidence has shown that upright and inverted faces are processed by common mechanisms; quantitative processing differences may result in the FIE. ${ }^{26-28}$ However, more recent research examined qualitative and quantitative processing differences for inverted faces vs upright faces recognition by using dynamic causal modeling and Bayesian model selection method in healthy participants. ${ }^{29}$ The results indicated that upright and inverted faces were processed by distinct qualitative and quantitative pathways, with upright face processing involving isolated activation of the OFA/FFA with concurrent lateral inhibition of the lateral occipital cortex, and inverted face processing involving increased couplings to the intraparietal sulcus without inhibition of the lateral occipital cortex. In addition, the extent of effective connectivity within the pathways from the visual cortex to OFA could predict individual differences in behavioral FIE.

Recent behavioral experiments yielded mixed results of inversion on face recognition among most individuals with autism spectrum disorders (ASD) ${ }^{30,31}$ However, the differences in the processing of inverted and upright faces and the mechanisms mediating FIE in patients with schizophrenia are still unclear. Face inversion is a well-established marker of holistic face processing, and could decrease the representativeness of facial identity and facial expressions. ${ }^{32,33}$ Numerous researches on 
configural face processing in healthy and schizophrenic subjects suggested that configural face processing is highly relevant to social functioning including memory and executive function. ${ }^{34}$ Face recognition may rely more on configural processing, however, inversion could affect configural face processing. ${ }^{35}$ Additionally, both upright and inverted faces are holistically processed, but inversion could lead to reduced overall processing efficiency. ${ }^{36}$ By designing this study, we aim to better understand the mechanisms under FIE in patients with schizophrenia and to investigate the differences in the underlying mechanisms between patients with schizophrenia and controls from the community. In the present study, we investigated the neural basis during upright and inverted faces processing in schizophrenia patients and community controls using fMRI to characterize diseasespecific face recognition markers. Using a task including images of upright, inverted, scrambled faces and objects, we contrasted the processing of upright and inverted faces, as well as their judgment of upright or inverted faces, compared to the judgment of scrambled or nonface object images. Our hypothesis is that, compared to community controls, schizophrenia patients would demonstrate abnormal processing of both upright and inverted faces.

\section{Materials and Methods Study Design}

The study followed a prospective, non-randomized, experimental, and controlled design. $G^{*}$ Power software was used to calculate the sample size of the study. The parameters are as follows: effect size: $0.8, \alpha=0.05$, and test power: 0.9. Thus, each group should include at least 28 subjects.

\section{Patients}

The study recruited 69 subjects, who were divided into two groups, with 34 patients in the first-episode schizophrenia (FSZ) group and 35 individuals in the healthy control (CON) group. All the subjects were recruited from the Affiliated Brain Hospital of Guangzhou Medical University (Guangzhou Huiai Hospital), Guangdong province. The diagnosis of schizophrenia was confirmed by experienced clinical psychiatrists using the Diagnostic and Statistical Manual of Mental Disorders, Fourth Edition criteria (DSM-IV).
All the participants were subject to strict inclusion management as the following criteria: 1) subjects aged 17 to 50 years at the time of diagnosis for both groups; 2) subjects in the patient group were diagnosed with schizophrenia for the first time, with an illness duration of no more than 2 years and had received antipsychotics for no more than one year before enrollment; 3 ) subjects received formal education for at least 6 years; 4) subjects in the control group were recruited from the community and did not meet the Criteria of Psychosis-risk Syndromes or DSM-IV for any mental disorders, and had no documented family history of psychiatric problems or medications. The demographic variables of age, gender and education were matched at the group level between the control group and the schizophrenia group.

The exclusion criteria for the subjects were 1) subjects who met DSM-IV criteria for any psychotic disorders, or had delirium, dementia, amnesia, or other severe cognitive impairments in the past or intellectual developmental disabilities $(\mathrm{IQ}<70)$ before their schizophrenia diagnosis was made; 2) patients with clinically significant somatic diseases; 3) individuals with substance abuse in the past 3 months; 4) patients with a documented history of brain injury, epilepsy, or other known diseases of central nervous system; 5) patients who had received MECT therapy in the past 6 months.

This study was conducted in accordance with the Declaration of Helsinki and was approved by the Institutional Review Board of the Affiliated Brain Hospital of Guangzhou Medical University. All the participants provided written informed consent. In detail, for subjects under 18 years of age, informed consent obtained from a parent or legal guardian of each subject.

\section{Cognitive and General Psychiatric Assessments}

All the participants completed a demographic questionnaire for us to obtain the demographic data such as age, gender, education level, marital status, body mass index (BMI), and family history of mental illness.

Psychotic symptoms were assessed in the schizophrenia group using the Positive and Negative Syndrome Scale (PANSS), ${ }^{37}$ applied by two trained psychiatrists or psychologists. The Personal and Social Performance Scale $(\mathrm{PSP})^{38}$ was used to test the social function of patients with schizophrenia. The antipsychotic medication status at the time of testing was recorded in Table 1, including 
Table I Demographic, Clinical and Cognitive Characteristics

\begin{tabular}{|c|c|c|c|c|c|}
\hline \multirow[t]{2}{*}{ Characteristics } & \multicolumn{2}{|c|}{ FSZ } & \multicolumn{2}{|c|}{ CON } & \multirow{2}{*}{$\begin{array}{l}\text { Statistic } \\
\mathrm{t}, \mathrm{df}, \mathrm{p}\end{array}$} \\
\hline & $\mathbf{n}$ & Mean (S.D.) & $\mathbf{n}$ & Mean (S.D.) & \\
\hline Age & 31 & $25.0(6.8)$ & 32 & $24.2(5.1)$ & $\mathrm{t}=0.536, \mathrm{df}=6 \mathrm{I}, p=0.594$ \\
\hline Gender (M/F) & 31 & $17 / 14$ & 32 & $16 / 16$ & Chi square $=0.148, p=0.802$ \\
\hline Year of education & 31 & II.I(3.3) & 32 & $11.7(3.1)$ & $\mathrm{t}=-0.692, \mathrm{df}=6 \mathrm{I}, p=0.492$ \\
\hline Married $(\mathrm{Y} / \mathrm{N})$ & 31 & $11 / 20$ & 32 & $8 / 24$ & \\
\hline Handedness (R/L) & 31 & $30 / 1$ & 32 & $30 / 2$ & \\
\hline Family history $(\mathrm{Y} / \mathrm{N})$ & 31 & $7 / 24$ & 32 & $0 / 32$ & \\
\hline Simple IQ & 30 & $96.1(13.2)^{* *}$ & 32 & $105.8(12.1)$ & $\mathrm{t}=3.015, \mathrm{df}=60, p=0.004$ \\
\hline Block diagram test & 31 & $8.9(2.7)^{* * *}$ & 32 & $11.4(2.5)$ & $\mathrm{t}=-3.729, \mathrm{df}=61, p=0.000$ \\
\hline Vocabulary & 30 & $9.7(2.7)$ & 32 & $10.7(2.2)$ & $\mathrm{t}=-1.564, \mathrm{df}=60, p=0.123$ \\
\hline RBANS total & 31 & $368.3(61.7)^{* * *}$ & 31 & $437.2(48.6)$ & $\mathrm{t}=-4.886, \mathrm{df}=60, p=0.000$ \\
\hline Immediate memory & 31 & $57.1(16.8)^{* * *}$ & 32 & $74.9(13.8)$ & $\mathrm{t}=-4.602, \mathrm{df}=6 \mathrm{I}, p=0.000$ \\
\hline Visual span & 31 & $76.6(13.6)^{* *}$ & 32 & $86.3(14.2)$ & $\mathrm{t}=-2.778, \mathrm{df}=6 \mathrm{I}, p=0.007$ \\
\hline Language & 31 & $71.8(19.8)^{* * *}$ & 32 & $88.7(\mid 5.8)$ & $\mathrm{t}=-3.753, \mathrm{df}=6 \mathrm{I}, p=0.000$ \\
\hline Attention & 31 & $92.7(17.9)^{*}$ & 31 & $103.2(13.7)$ & $\mathrm{t}=-2.577, \mathrm{df}=60, p=0.012$ \\
\hline Delayed memory & 31 & $70.1(17.9)^{* * *}$ & 32 & $85.3(10.9)$ & $\mathrm{t}=-4.079, \mathrm{df}=6 \mathrm{I}, p=0.000$ \\
\hline TMT-A & 31 & $52.3(19.7)^{* *}$ & 32 & $38.8(13.5)$ & $\mathrm{t}=-3.175, \mathrm{df}=61, p=0.004$ \\
\hline MSCEIT & 31 & $80.3(9.6)$ & 32 & $83.9(6.8)$ & $\mathrm{t}=\mathrm{I} .747, \mathrm{df}=6 \mathrm{I}, p=0.086$ \\
\hline TEPS total & 29 & $67.8(11.7)$ & 32 & $72.9(9.5)$ & $\mathrm{t}=-1.883, \mathrm{df}=59, p=0.065$ \\
\hline SHAPS total & 31 & $30.4(5.3)$ & 32 & $33.0(5.2)$ & $\mathrm{t}=-1.984, \mathrm{df}=6 \mathrm{I}, p=0.052$ \\
\hline BMI & 31 & $20.7(3.1)$ & 32 & $21.2(4.2)$ & $\mathrm{t}=-0.502, \mathrm{df}=61, p=0.618$ \\
\hline Duration of illness (months) & 31 & $8.5(7.0)$ & & & \\
\hline Age of first episode & 31 & $24.0(6.8)$ & & & \\
\hline Medication status at time of testing & & & & & \\
\hline None medicated & 8 & & & & \\
\hline Antipsychotic & 20 & & & & \\
\hline Atypical & 18 & & & & \\
\hline Atypical+Traditional & 2 & & & & \\
\hline Antidepression & 1 & & & & \\
\hline Unknown medication & 2 & & & & \\
\hline Duration of medication treatment (days) & 31 & $26.4(37.2)$ & & & \\
\hline Chlorpromazine Equivalents (mg) & 31 & $222.1(244.4)$ & & & \\
\hline PANSS total & 31 & $65.6(15.0)$ & & & \\
\hline PANSS positive & 31 & $17.6(5.1)$ & & & \\
\hline PANSS negative & 31 & 13.7(4.1) & & & \\
\hline PANSS general & 31 & $34.3(8.6)$ & & & \\
\hline PSP total & 31 & $55.4(15.3)$ & & & \\
\hline Past hospitalization history (Y/N) & 31 & $3 / 28$ & & & \\
\hline Auditory hallucination $(\mathrm{Y} / \mathrm{N})$ & 31 & $20 / 11$ & & & \\
\hline
\end{tabular}

Notes: $* p<0.05, * * p<0.01, * * * p<0.001$

Abbreviations: FSZ, patients with first-episode schizophrenia; CON, healthy controls; MSCEIT, Mayer-Salovey-Caruso Emotional Intelligence Test; RBANS, the Repeatable Battery for the Assessment of Neuropsychological Status; TMT-A, Trail-Making Test: Part A; TEPS, the Temporal Experience of Pleasure Scale; SHAPS, the Snaith-Hamilton Pleasure Scale; PANSS, the positive and negative syndrome scale; PSP, Personal and Social Performance; S.D., standard deviation.

types, duration and chlorpromazine equivalent doses of the medications. We also assessed all the patients for auditory hallucination.

We applied the Repeatable Battery for the Assessment of Neuropsychological Status (RBANS), ${ }^{39}$ Trail-Making Test: Part A (TMT-A) and MayerSalovey-Caruso Emotional Intelligence Test:
Managing Emotions (MSCEIT) $)^{40}$ tests in the MATRICS Consensus Cognitive Battery (MCCB), ${ }^{41,42}$ and block diagram test and vocabulary test were used to evaluate the cognitive performance of each subject. The cognitive evaluation was carried out by a trained and certified psychiatrist, psychologist or neuropsychological examiner. 
The Temporal Experience of Pleasure Scale (TEPS) ${ }^{43}$ and the Snaith-Hamilton Pleasure Scale (SHAPS) ${ }^{44}$ were used to assess the anhedonia experience in patients with schizophrenia. The relationship between anhedonia and features of schizophrenia was also examined.

\section{Stimuli}

Stimuli consisted of grayscale images of upright and inverted faces with neutral expression, and happy faces, provided by Chinese Facial Affective Picture System (CFAPS) ${ }^{45}$ as well as grayscale images of scrambled faces and objects. The scrambled faces were created by randomly mixing the neutral faces into squares of $10 \times 10$ pixel grids. All images subtended at $6.2 \times 6.2^{\circ}$ of the visual angles and were presented at the center of the screen, with the mean brightness normalized (ie, the images were equalized in terms of brightness). We adopted a blocked design containing five categories displayed in the following sequence: upright faces, objects, inverted faces, scrambled faces and happy faces.

\section{Experimental Procedure}

For each run, block trial began with a white point displayed at the center of the black screen, followed by image displays containing a sequence of upright faces, objects, inverted faces, scrambled faces, and happy faces that appeared at a rate of $2 \mathrm{~Hz}$ in blocks of $12 \mathrm{~s}$, with $12 \mathrm{~s}$ of blank interval. Each image stimulus was presented for 300 $\mathrm{ms}$, followed by a $200-\mathrm{ms}$ interstimulus interval per block. The sum of the duration of fixation and sample displays was constant. Each 12s block presented 24 images per stimulus type, and the images were randomly selected from the same category type, with 30 images in each category. Faces image samples included female and male faces at a ratio of 1:1. According to the study protocol, each block type was presented for a total of $252 \mathrm{~s}$ per run. Subjects performed a one-back task during which they were asked to press a key to identify the same images (see Figure 1 for the illustration of the procedure, with samples provided).

\section{MRI Acquisition}

A 3.0-T Philips Achieva MRI scanner (Philips, Netherlands) was used to obtain data of blood oxygenation level-dependent cortical activity. High-resolution fMRI brain images were obtained with the following parameters: repetition time $(\mathrm{TR})=2000 \mathrm{~ms}$, echo time $(\mathrm{TE})=30 \mathrm{~ms}$, field of view $=220 \times 220 \mathrm{~mm}^{2}$, voxel size $=3.44 \mathrm{~mm} \times$



Figure I An illustration of the procedure. For each run, images sample displays containing a sequence of upright faces, objects, inverted faces, scrambled faces, and happy faces that appeared at a rate of $2 \mathrm{~Hz}$ in blocks of $12 \mathrm{~s}$ and with $12 \mathrm{~s}$ blank blocks interval at a time. Each image stimulus was presented for $300 \mathrm{~ms}$, followed by a $200 \mathrm{~ms}$ interstimulus interval per block. The sum of the duration of fixation and sample displays was constant. Each block presented 24 images per stimulus type and lasted I2s. These images were randomly selected from the same category type. Each category contains 30 images. Faces images sample included female and male faces on each half.

$3.44 \mathrm{~mm}$, matrix size $=64 \times 64$, number of slices $=33$, and slice thickness $=4 \mathrm{~mm}$. A total of 126 functional fullbrain volumes were collected per subject in each run.

Stimuli were presented with the Psychtoolbox 3.0 for MATLAB (http://psychtoolbox.org), and projected onto a screen viewed by the participants through a mirror fixed in the MRI scanner. All the subjects were instructed to keep their eyes opened, look at the pictures of the oneback task, and remain still in a supine position during the whole scanning. The MRI data were acquired at the Affiliated Brain Hospital of Guangzhou Medical University on the same day for clinical and cognitive assessments.

\section{Statistical Analysis}

\section{Demographic, Clinical and Cognitive Data}

The statistical analyses were performed with SPSS 23 software. Differences in demographic data (age, gender, education), clinical data and cognitive data were compared between groups using independent samples $t$-test and Chi square test; post-hoc test was applied as needed. Finally, Pearson correlations test was performed to compare the fMRI data and patient performance (clinical symptoms, medication dosages, and cognitive function). Benjamini 
\& Hochberg (BH) corrected significance level (at $\alpha=0.05$ ) was used as the protected correction level for correlations.

\section{Preprocessing of fMRI Data}

The fMRI data was analyzed using the general linear model for block designs in statistical parametric mapping version 12 (SPM12) software (the Wellcome Department of Imaging Neuroscience, London, UK) implemented in MATLAB (MathWorks). Functional images were preprocessed with correction of slice acquisition time, head motion correction (realignment), co-registration, and spatial normalization by using $\mathrm{T} 1$ image unified segmentation and resampled to voxel size of $3 \times 3 \times 3 \mathrm{~mm}^{3}$. Subsequently, the functional images underwent spatial smoothing with $8 \times 8 \times 8 \mathrm{~mm}^{3}$ full-width at half-maximum (FWHM) Gaussian kernel. Subjects with a head motion of more than $2.5 \mathrm{~mm}$ and with an angular motion of more than $2^{\circ}$ across the whole scan were excluded.

\section{First-Level and Second-Level Analysis}

In the first-level analysis, the design matrix consisted of five regressors of interest, each representing the upright faces, objects, inverted faces, scrambled faces, and happy faces, with same display duration convolved with a canonical hemodynamic response function (HRF). Six head motion parameters were defined in the matrix as effects of no interest. Finally, a GLM HRF model was calculated for each subject. Within the FSZ or CON group, the upright face associated activation effect was defined using conjunctions of t-contrasts including upright faces $>$ inverted faces, upright faces $>$ scramble faces, and upright faces $>$ objects. The inverted face associated activation effect was defined using conjunctions of t-contrasts including inverted faces $>$ upright faces, inverted faces $>$ scramble faces, and inverted faces $>$ objects.

To further confirm the difference of brain activation between the FSZ group and the CON group, a second level two-sample $t$ test was performed, with a threshold of significant difference being $p<0.05$ (false discovery rate (FDR) corrected, performed in DPABI version 4.0 $)^{46}$ and a cluster threshold being no less than 10 voxels. Intergroup differences during each condition were tested with the contrast of CON vs FSZ. Firstly, we established the CON vs FSZ contrast during displays of upright faces, objects, inverted faces, scrambled faces, and happy faces. Then, we defined CON vs FSZ inter-group associated upright face activation effect (including t-contrast conjunctions of upright faces $>$ inverted faces, upright faces $>$ scramble faces, and upright faces $>$ objects) and inverted face activation effect (including t-contrast conjunctions of inverted faces $>$ upright faces, inverted faces $>$ scramble faces, and inverted faces $>$ objects). We then masked the corrected SPM with a contrast-specific brain mask to limit findings to task activated regions for inter-group analysis. This mask was the combination of the activated voxels discovered in one-sample analysis in healthy controls and patients with schizophrenia under each contrast.

\section{Selection of Region of Interest (ROI)}

Only voxels that reached the significance level for each contrast of inter-group analysis $(p<0.05$, FDR corrected) were included in ROIs. We defined category-selective ROIs from contrasts between conditions of upright faces, inverted faces, scrambled faces, and objects. Upright face selective areas were extracted the t-contrast maps regarding conjunctions of upright faces $>$ inverted faces, upright faces $>$ scrambled faces, and upright faces $>$ objects $(p<$ 0.05, FDR corrected). Similarly, inverted-face selective areas were extracted from t-contrast maps regarding conjunctions of inverted faces $>$ upright faces, inverted faces $>$ scrambled faces, and inverted faces $>$ objects $(p<0.05$, FDR corrected). These ROIs were identified as spheres with a radius of $6 \mathrm{~mm}$ centered around the peak voxel of each activated cluster, and eigenvariate of the entire ROI region was extracted using the toolbox in SPM version 12 (see Table 5 for ROI information).

\section{Results}

\section{Demographic, Clinical and Cognitive Results}

Data from six subjects were excluded of the final analysis, including one who withdrew from the study, two who failed to finish non-imaging tests, and three with excessive head motion during the whole scan. A total of 63 subjects completed the behavioral experiments for analysis. Therefore, this data set included $31 \mathrm{FSZ}$ patients and 32 CON subjects.

Demographic (age, gender, years of education, and medication status at the time of testing), and clinical data (PANSS symptom ratings) are presented in Table 1. There were no significant age, gender or education differences between the two groups. The patients with FSZ presented a disease duration of 8.5 months in average, and their doses of antipsychotics were converted to equivalent chlorpromazine dosage (CPZ). Cognitive performances are listed in Table 1. Patients with schizophrenia showed 
clear deficits in TMT-A test and all the RBANs tests, while no difference from the controls was observed in the MSCEIT test. Additionally, patients also showed no differences in TEPS and SHAPS scores compared to healthy controls.

\section{Neuroimaging Results \\ Conjunction Results of FIE}

The conjunction analyses of upright and inverted face related contrasts of CON (see details in Table 3) and FSZ (see details in Table 2) revealed significant activation in various clusters comprising brain regions including fusiform, middle occipital gyrus, and inferior occipital gyrus, suggesting great activation in the FIE networks across groups.

(Tables 2 and 3 about here)

\section{Greater Functional Activities in the CON Group} Inter-group differences of upright face selective areas showed lower neural responses in the left parahippocampus, left hippocampus, left limbic lobe, left middle occipital gyrus, and left cuneus in patients with schizophrenia (see details in Table 4, and Figure 2A and C). Inter-group differences of inverted face selective areas showed lower neural responses in the left inferior parietal gyrus and left postcentral gyrus in the FSZ patients (see details in Table 4 and Figure 2B).

\section{Greater Functional Activities in the FSZ Group} Inter-group differences of upright face selective areas showed that higher neural responses were observed in left inferior parietal gyrus, left postcentral gyrus, right postcentral gyrus, and right parietal lobe in patients with schizophrenia, while no significant difference was observed in inverted face selective areas between patients and controls (see details in Table 4 and Figure 2C).

\section{ROls for Correlation}

Four ROIs were defined from the contrasts between the four face conditions (Table 5). A conjunction analysis using a conjunction-null hypothesis ${ }^{47}$ was performed to identify common areas of activation across contrasts of upright faces $>$ scrambled faces, upright faces $>$ object, upright faces $>$ inverted faces, and contrasts of inverted faces $>$ scrambled faces, inverted faces $>$ object, inverted faces $>$ upright faces. According to the results, upright face processing was related to the left parahippocampus and left middle occipital gyrus, while inverted face processing was related to the left postcentral and inferior parietal lobule.

\section{Functional Activity - Correlation with Cognitive and} Clinical Performance

In the FSZ group, the reduced functional activity of the left middle occipital gyrus showed negative correlation with PSP total performance $(r=-0.495, p=0.005$, two tailed, see details in Table 6 and Figure 3) and positive correlation with PANSS general scores $(r=0.396, p=$ 0.05, two tailed, but failed the Benjamini and Hochberg correction, see details in Table 6), while the functional activity of the left postcentral gyrus was significantly associated with block diagram test scores $(r=0.431, p=$ 0.016, two tailed, see details in Table 6 and Figure 3). (see all details in Table 6).

\section{Discussion}

To our knowledge, the present study is the first to investigate the mechanisms underlying FIE in patients with first-episode schizophrenia. Our results suggest a distinct functional activity for upright and inverted face processing in FSZ patients, which located within the cortical face and non-face networks. According to our data, the patients with FSZ processed both inverted faces and upright faces less efficiently than controls; and for the patients, inverted faces are processed less efficiently than upright faces. Upright face recognition in schizophrenia patients was associated with activated face-selective network, including the fusiform, inferior occipital gyrus, and superior temporal gyrus. Non-face-specific areas were also activated in the patients, including the insula, precuneus, middle and inferior temporal gyrus, inferior and superior parietal lobule, middle occipital gyrus, and superior and middle frontal gyrus. On the other hand, inverted face recognition was not associated with activated fusiform and temporal face-selective areas, as well as the insula, parietal, and temporal non-face-selective brain regions. Inverted face recognition has induced slight and isolated activation of the occipital and frontal regions.

We concluded that turning faces upside down significantly decreased or suppressed the neural response of some face-processing related brain regions, especially the fusiform and the occipital face area (OFA), and temporal and parietal non-face selective areas. However, how the activation of these non-face-specific areas occurred is still unclear. Upright faces are identified in a holistic pathway, whereas inverted facial features are identified in a fragmented manner. Conversely, other findings predicted by an optimal Bayesian integrator study showed that whole faces were not recognized better than the sum of their components showed 
Table 2 Significantly Activated Regions During the Face Inversion Task in Patients with Schizophrenia

\begin{tabular}{|c|c|c|c|c|c|}
\hline \multirow[t]{2}{*}{ Regions } & \multirow[t]{2}{*}{ Cluster Size } & \multirow[t]{2}{*}{$\mathbf{T}$} & \multicolumn{3}{|c|}{ MNI Coordinates } \\
\hline & & & $x$ & $\mathbf{Y}$ & $\mathbf{Z}$ \\
\hline $\begin{array}{l}\text { Conjunction (upright >inverted, upright >scrar } \\
\text { Upright >inverted } \\
\text { Cluster I }\end{array}$ & & & & & \\
\hline $\begin{array}{l}\text { Right insula } \\
\text { Upright >scramble } \\
\text { Cluster I }\end{array}$ & 16 & 3.84 & 36 & 12 & 12 \\
\hline $\begin{array}{l}\text { Right temporal lobe/fusiform gyrus } \\
\text { Cluster } 2\end{array}$ & 912 & 8.55 & 42 & -48 & -21 \\
\hline $\begin{array}{l}\text { Left temporal lobe/fusiform gyrus } \\
\text { Cluster } 3\end{array}$ & 607 & 8.51 & -42 & -48 & -21 \\
\hline $\begin{array}{l}\text { Left insula } \\
\text { Cluster } 4\end{array}$ & 688 & 6.52 & -30 & 21 & 3 \\
\hline $\begin{array}{l}\text { Right insula } \\
\text { Cluster } 5\end{array}$ & 1013 & 7.70 & 33 & 21 & 6 \\
\hline $\begin{array}{l}\text { Right superior parietal lobule } \\
\text { Cluster } 6\end{array}$ & 674 & 5.95 & 30 & -57 & 51 \\
\hline $\begin{array}{l}\text { Left inferior parietal lobule/postcentral lobule } \\
\text { Cluster } 7\end{array}$ & 736 & 6.87 & -42 & -33 & 48 \\
\hline $\begin{array}{l}\text { Left cingulate gyrus } \\
\text { Upright >objects } \\
\text { None }\end{array}$ & 525 & 6.73 & -6 & 12 & 45 \\
\hline $\begin{array}{l}\text { Conjunction (inverted >upright, inverted >scra } \\
\text { Inverted > upright } \\
\text { Cluster I }\end{array}$ & & & & & \\
\hline $\begin{array}{l}\text { Right middle temporal gyrus/middle occipital gyrus } \\
\text { Cluster } 2\end{array}$ & 97 & 4.25 & 45 & -69 & 27 \\
\hline $\begin{array}{l}\text { Left middle occipital gyrus } \\
\text { Cluster } 3\end{array}$ & 98 & 4.69 & -39 & -78 & 27 \\
\hline $\begin{array}{l}\text { Left middle frontal gyrus } \\
\text { inverted >scramble } \\
\text { Cluster I }\end{array}$ & 155 & 5.50 & -21 & 33 & 39 \\
\hline $\begin{array}{l}\text { Left inferior occipital gyrus } \\
\text { Cluster } 2\end{array}$ & 445 & 6.77 & -42 & -78 & -12 \\
\hline $\begin{array}{l}\text { Right middle occipital gyrus/inferior occipital gyrus } \\
\text { Cluster } 3\end{array}$ & 681 & 7.91 & 42 & -72 & -12 \\
\hline $\begin{array}{l}\text { Left inferior frontal gyrus } \\
\text { Cluster } 4\end{array}$ & 577 & 5.23 & -33 & 21 & 9 \\
\hline $\begin{array}{l}\text { Right insula/inferior frontal gyrus } \\
\text { Cluster } 5\end{array}$ & 84 & 4.28 & 30 & 24 & 0 \\
\hline $\begin{array}{l}\text { Right inferior frontal gyrus/precentral gyrus } \\
\text { Cluster } 6\end{array}$ & 604 & 5.50 & 42 & 0 & 36 \\
\hline $\begin{array}{l}\text { Left medial frontal gyrus/cingulate gyrus } \\
\text { Cluster } 7\end{array}$ & 438 & 6.07 & 0 & 12 & 48 \\
\hline $\begin{array}{l}\text { Left inferior parietal gyrus } \\
\text { Cluster } 8\end{array}$ & 585 & 5.16 & -27 & -42 & 45 \\
\hline $\begin{array}{l}\text { Right superior parietal lobule/inferior parietal lobule } \\
\text { Inverted > objects } \\
\text { None }\end{array}$ & 607 & 5.45 & 30 & -57 & 51 \\
\hline
\end{tabular}

Notes: The level of significance was set using a threshold of $\alpha=0.05$ false discovery rate, FDR whole brain corrected. Abbreviation: MNI, Montreal Neurological Institute. 
Table 3 Significantly Activated Regions During the Face Inversion Task in Healthy Controls

\begin{tabular}{|c|c|c|c|c|c|}
\hline \multirow[t]{2}{*}{ Regions } & \multirow[t]{2}{*}{ Cluster Size } & \multirow[t]{2}{*}{$\mathbf{T}$} & \multicolumn{3}{|c|}{ MNI Coordinates } \\
\hline & & & $\mathbf{x}$ & $\mathbf{Y}$ & $\mathbf{Z}$ \\
\hline $\begin{array}{l}\text { Conjunction (upright >inverted, upright >scr } \\
\text { Upright >inverted } \\
\text { Cluster I }\end{array}$ & & & & & \\
\hline $\begin{array}{l}\text { Left temporal lobe/hippocampus } \\
\text { Cluster } 2\end{array}$ & 142 & 5.37 & -33 & -48 & 0 \\
\hline $\begin{array}{l}\text { Left occipital lobe/cuneus } \\
\text { Upright >scramble } \\
\text { Cluster I }\end{array}$ & 152 & 3.93 & -9 & 90 & 3 \\
\hline $\begin{array}{l}\text { Right temporal lobe/fusiform gyrus } \\
\text { Cluster } 2\end{array}$ & 2034 & 10.14 & 42 & -51 & -24 \\
\hline $\begin{array}{l}\text { Right inferior frontal gyrus/middle frontal gyrus } \\
\text { Cluster } 3\end{array}$ & 1144 & 7.02 & 48 & 18 & 6 \\
\hline $\begin{array}{l}\text { Left insula/inferior frontal gyrus } \\
\text { Cluster } 4\end{array}$ & 486 & 5.41 & -33 & 21 & 0 \\
\hline $\begin{array}{l}\text { Left inferior parietal lobule } \\
\text { Cluster } 5\end{array}$ & 161 & 4.83 & -27 & -57 & 39 \\
\hline $\begin{array}{l}\text { Right inferior parietal lobule } \\
\text { Cluster } 6\end{array}$ & 338 & 5.41 & 39 & -51 & 45 \\
\hline $\begin{array}{l}\text { Right medial frontal gyrus } \\
\text { Upright >objects } \\
\text { None }\end{array}$ & 309 & 5.20 & 6 & 18 & 51 \\
\hline $\begin{array}{l}\text { Conjunction (inverted >upright, inverted >se } \\
\text { Inverted >upright } \\
\text { Cluster I }\end{array}$ & & & & & \\
\hline $\begin{array}{l}\text { Right precuneus/middle occipital lobule } \\
\text { Inverted >scramble } \\
\text { Cluster I }\end{array}$ & 136 & 4.29 & 33 & -81 & 33 \\
\hline $\begin{array}{l}\text { Right middle occipital gyrus } \\
\text { Cluster } 2\end{array}$ & 953 & 9.42 & 42 & -72 & -12 \\
\hline $\begin{array}{l}\text { Left inferior occipital gyrus/middle occipital gyrus } \\
\text { Cluster } 3\end{array}$ & 801 & 9.30 & -39 & -78 & -12 \\
\hline $\begin{array}{l}\text { Right middle temporal gyrus } \\
\text { Cluster } 4\end{array}$ & 183 & -5.12 & 60 & 3 & -12 \\
\hline $\begin{array}{l}\text { Right occipital lobule/cuneus } \\
\text { Cluster } 5\end{array}$ & 3430 & -7.40 & 6 & -90 & 21 \\
\hline $\begin{array}{l}\text { Left insula } \\
\text { Cluster } 6\end{array}$ & 161 & 5.28 & -33 & 24 & 0 \\
\hline $\begin{array}{l}\text { Left middle frontal gyrus } \\
\text { Cluster } 7\end{array}$ & 891 & -6.08 & -21 & 36 & 45 \\
\hline $\begin{array}{l}\text { Right insula } \\
\text { Cluster } 8\end{array}$ & 1030 & 5.99 & 33 & 24 & 3 \\
\hline $\begin{array}{l}\text { Left precentral gyrus } \\
\text { cluster } 9\end{array}$ & 297 & 5.18 & -39 & -3 & 39 \\
\hline $\begin{array}{l}\text { Right medial frontal gyrus } \\
\text { cluster } 10\end{array}$ & 524 & 6.48 & 9 & 15 & 51 \\
\hline $\begin{array}{l}\text { Right inferior parietal lobule } \\
\text { Cluster II }\end{array}$ & 562 & 5.67 & 33 & -51 & 45 \\
\hline $\begin{array}{l}\text { Left parietal lobe/precuneus } \\
\text { Inverted >objects } \\
\text { None }\end{array}$ & 359 & 5.55 & -24 & -60 & 45 \\
\hline
\end{tabular}

Notes: The level of significance was set using a threshold of $\alpha=0.05$ false discovery rate, FDR whole brain corrected. Abbreviation: MNI, Montreal Neurological Institute. 
Table 4 Significantly Activated Regions During the Face Inversion Task Between Patients with Schizophrenia and Healthy Controls

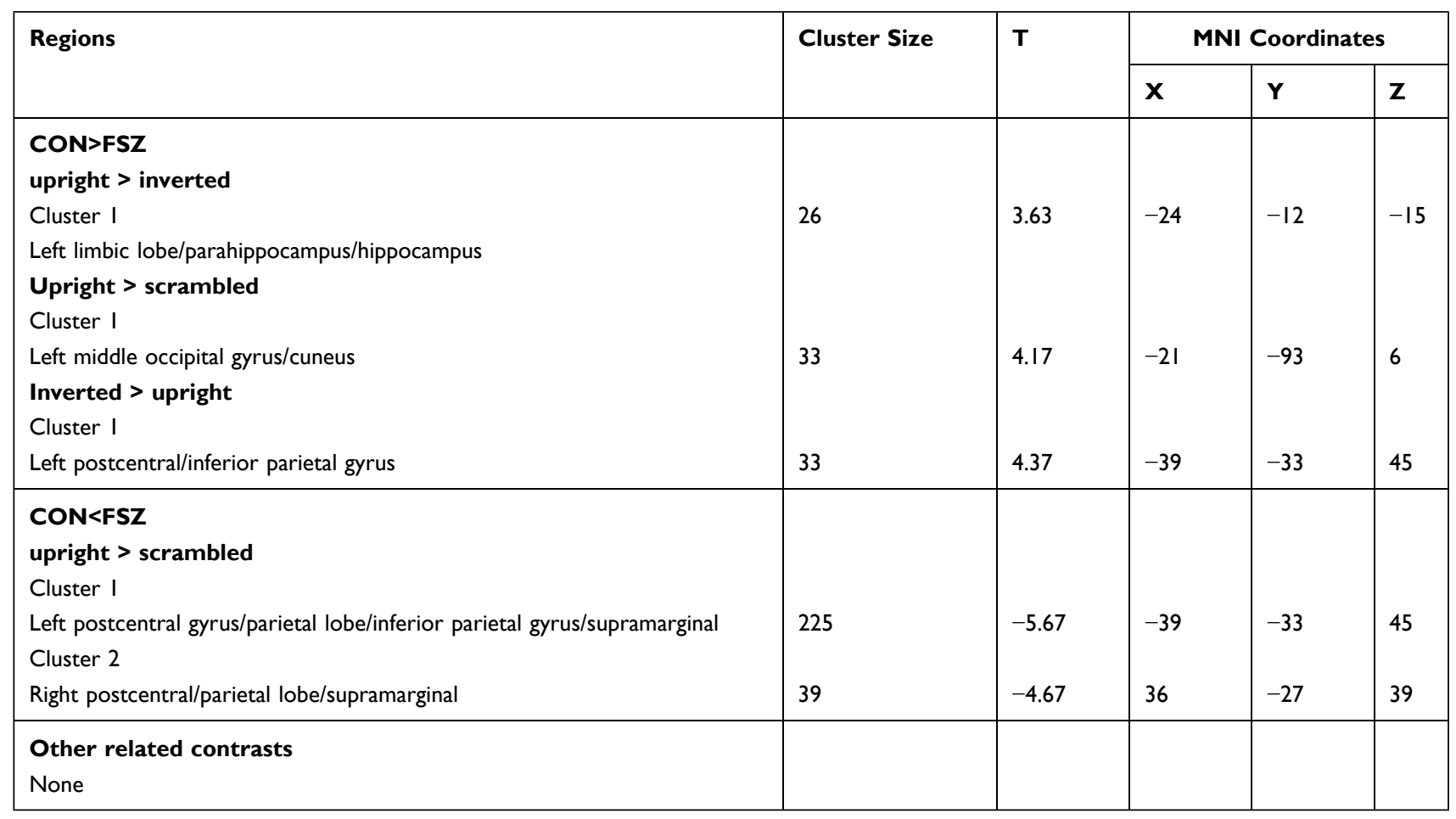

Note: The level of significance was set using a threshold of $\alpha=0.05$ false discovery rate, FDR contrast-specific brain mask corrected.

Abbreviations: MNI, Montreal Neurological Institute; CON, healthy controls; FSZ, patients with first-episode schizophrenia.

Table 5 Three ROls Selected for Correlations

\begin{tabular}{|l|l|l|l|l|l|}
\hline Regions & \multirow{2}{*}{$\begin{array}{l}\text { Cluster } \\
\text { Size }\end{array}$} & T & \multicolumn{3}{|c|}{ MNI Coordinates } \\
\cline { 3 - 6 } & & X & Y & Z \\
\hline $\begin{array}{l}\text { CON>FSZ } \\
\text { Upright>inverted } \\
\text { Left parahippocampus } \\
\text { Inverted>upright } \\
\text { Left postcentral/inferior } \\
\text { parietal gyrus } \\
\text { CON<FSZ }\end{array}$ & 33 & 3.63 & -24 & -12 & -15 \\
$\begin{array}{l}\text { Upright>scramble } \\
\text { Left middle occipital gyrus }\end{array}$ & 33 & 4.37 & -39 & -33 & 45 \\
\hline
\end{tabular}

Abbreviations: MNI, Montreal Neurological Institute; CON, healthy controls; FSZ, patients with first-episode schizophrenia.

separately. ${ }^{48,49}$ Still, other findings implied that inversion induces quantitative, rather than qualitative changes in the face recognition process. Upright features are, therefore, integrated more accurately and faster than inverted features. Face inversion disrupted the patient's ability to extract associated information when discriminating individual faces, and led to a significantly decreased response in recognition of faces. ${ }^{50}$ Our results showed a reduced response in the cortical face network during recognition of inverted faces, which was consistent with previous findings. Conclusively, these findings might suggest that inverted faces are processed similarly as upright faces, but inversion could lead to lower overall processing efficiency. ${ }^{51}$

Previous studies have examined the differences between the processing of upright and inverted faces in healthy controls. However, there is few available data regarding this process in individuals with major psychosis. Some behavioral experiments in individuals with ASD suggested a normal effect of inversion on face recognition. $^{30,31}$ A recent review summarized how the upright and inverted faces were processed and identified in individuals with ASD. ${ }^{52}$ Our findings about processing differences for upright faces vs inverted faces in patients with schizophrenia are consistent with those ASD studies, suggesting that quantitative processing differences might be critical for how the upright and inverted faces are processed in patients with schizophrenia.

We also found that both processing for upright and inverted faces were impaired in patients with schizophrenia. For inverted faces processing, patients with schizophrenia showed reduced neural response only in the left inferior parietal gyrus and left postcentral gyrus; while, for upright face processing, more regions exhibited abnormal functional activity. Together, these results showed that 
A

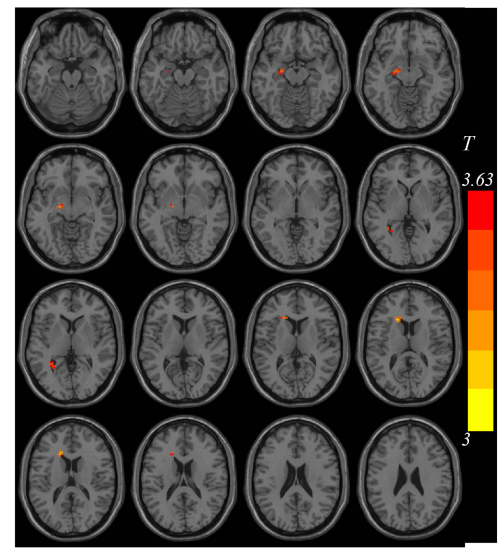

B

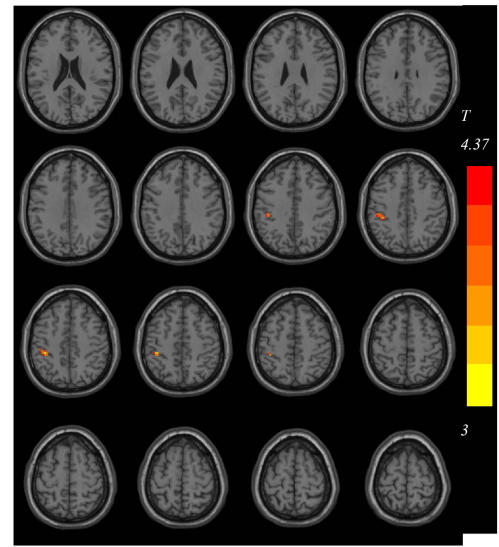

C

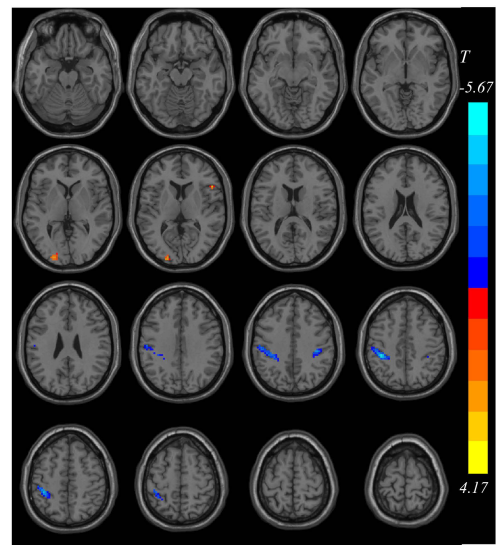

Figure 2 (A) Functional activity differences of upright selective areas between patients with FES and controls (CON>FES). The color bar represents the $t$ values of the functional activity group analysis. (B) Functional activity differences of upright selective areas between patients with FES and controls (CON<FES). The color bar represents the $t$ values of the functional activity group analysis. (C) Functional activity differences of inverted selective areas between patients with FES and controls (CON>FES). The color bar represents the $t$ values of the functional activity group analysis.

individuals with schizophrenia processed faces less efficiently than controls, and indicated that the impairment in upright face recognition was more severe and broader than in inverted face recognition in patients with schizophrenia.

There is evidence that regions for scene and object recognition, ie, the parahippocampal place area (PPA) and lateral occipital cortex, are both involved in the processing of inverted faces. ${ }^{53}$ However, in this present study, we observed reduced response in the hippocampus in processing upright faces vs inverted faces, and reduced response in the middle occipital gyrus in the upright face processing. We also found reduced response in the inferior parietal gyrus in processing inverted faces vs upright faces in patients with schizophrenia. These findings provided a clue for the mechanism mediating the processing of upright and inverted features in patients with schizophrenia, which might be the aberrant activation of non-face-selective areas rather than abnormal activation of face-specific areas.

Table 6 Correlations Between Face Processing and Cognitive, Clinical Performances

\begin{tabular}{|l|l|l|l|}
\hline Items & $\begin{array}{l}\text { PSP } \\
\text { Total }\end{array}$ & $\begin{array}{l}\text { PANSS } \\
\text { General }\end{array}$ & $\begin{array}{l}\text { Block } \\
\text { Diagram } \\
\text { Test }\end{array}$ \\
\hline $\begin{array}{l}\text { Left postcentral/inferior } \\
\text { parietal lobule } \\
\text { Left middle occipital } \\
\text { gyrus }\end{array}$ & -0.003 & -0.007 & $0.431^{*}$ \\
\hline
\end{tabular}

Notes: A: Benjamini and Hochberg corrected $p<0.05 / 2=0.025$ significant; ${ }^{*} p<0.05$, $* * p<0.005$.

Abbreviations: PANSS, the positive and negative syndrome scale; PSP, Personal and Social Performance.
Additionally, our results showed that the reduced activity in the left middle occipital gyrus for upright processing was correlated with the performance of PSP, which reflected the social function outcomes for this devastating illness. One previous study reported functional activity in the occipital cortex, showing a correlation between poor social adjustment and impaired social cognition in patients with chronic schizophrenia. ${ }^{54}$ Such findings are in line with the hypothesis that social functioning deficit may correlate with dysfunction in the processing of face information. We failed to find any correlation between inverted face processing and functional outcomes. As compromised inverted face processing could reduce the overall efficiency of face processing, ${ }^{36}$ it may play a medicating role in the functional outcome of patients. Our findings have provided evidence for the neural pathway that might be involved in determining the functional outcomes of this disorder.

\section{Conclusion}

We have demonstrated the abnormal networks involved in upright and inverted face processing in patients with firstepisode schizophrenia. Firstly, our results suggested that patients with FSZ process inverted faces and upright faces with similar performance, though inverted faces are processed a bit less efficiently than upright faces. Secondly, our data showed that upright face recognition impairment was more severe and broader than inverted face recognition in patients with schizophrenia. This shed light on the mechanism mediating the processing of upright and inverted features, which might be the aberrant activation of non-face-selective areas, rather than abnormal 

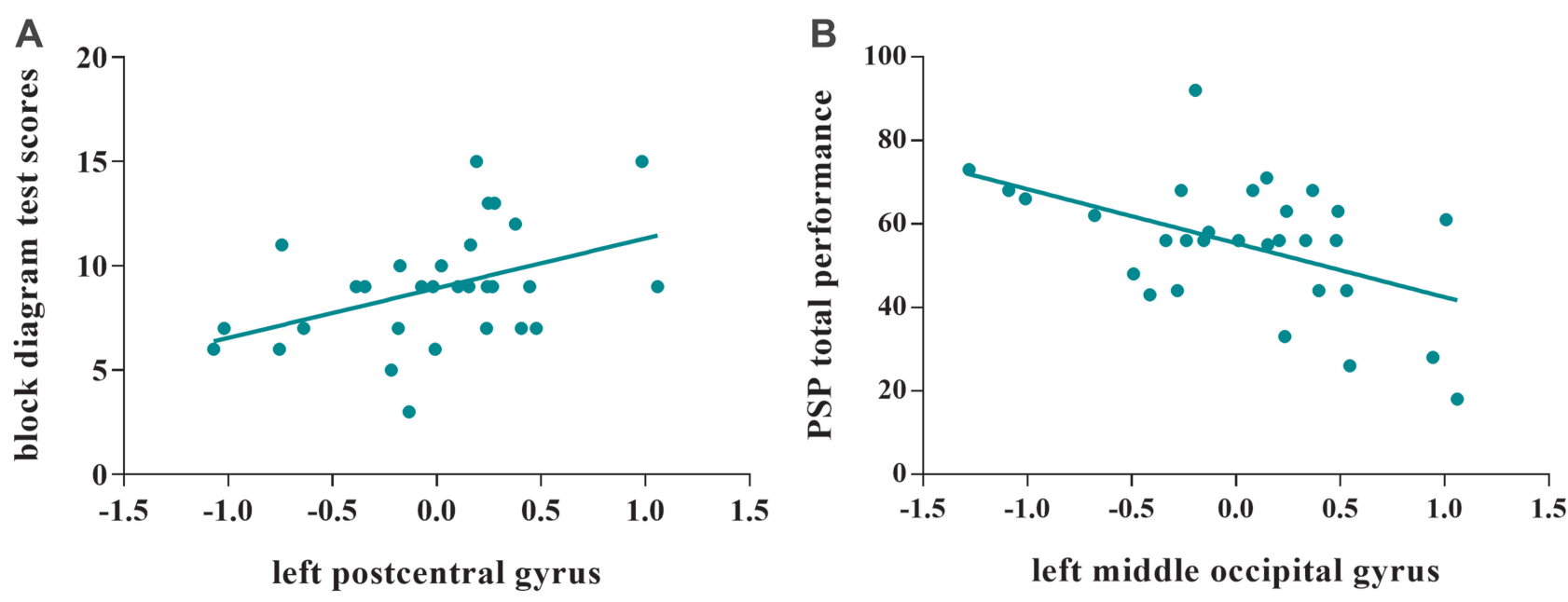

Figure 3 Correlations between the functional activity of the upright and inverted selective areas with cognitive and clinical performance. (A) represent the significant correlation between functional activity of the inverted selective areas and the scores of block diagram test. (B) represent the significant correlation between functional activity of the upright selective areas and the PSP total scores.

Abbreviation: PSP, The Personal and Social Performance Scale.

activation of face-specific areas. Furthermore, reduced neural response for upright face processing in the left middle occipital gyrus was correlated with social function outcomes, whereas no correlation was found between regions involved in inverted face processing. Therefore, the dysfunction of upright face processing rather than inverted face recognition might play a critical role in determining the functional outcome of this devastating disorder.

\section{Acknowledgments}

The authors would like to acknowledge the National Natural Science Foundation of China, the Guangzhou Science, Technology and Innovation Commission of Guangdong Province of China and Postdoctoral Scientific Research Start-up Foundation from The Affiliated Brain Hospital of Guangzhou Medical University of China for the financial support.

\section{Author Contributions}

All authors made a significant contribution to the work reported, whether that is in the conception, study design, execution, acquisition of data, analysis and interpretation, or in all these areas; took part in drafting, revising or critically reviewing the article; gave final approval of the version to be published; have agreed on the journal to which the article has been submitted; and agree to be accountable for all aspects of the work.

\section{Funding}

National Natural Science Foundation of China (grant 81671334) to Dr. Yingjun Zheng, Planed Science and Technology Projects of Guangzhou (grant 201904010267) to Dr. Yingjun Zheng, Planed Science and Technology Projects of Guangzhou (grant 2060404) to Mr. Shenglin She, Postdoctoral Science Research Foundation of Guangzhou (grant 520101-0201156) to Dr. Yi Liu.

\section{Disclosure}

The authors declare no conflicts of interest.

\section{References}

1. Bortolon C, Capdevielle D, Salesse RN, Raffard S. Self-face recognition in schizophrenia: an eye-tracking study. Front Hum Neurosci. 2016;10:3. doi:10.3389/fnhum.2016.00003

2. Lee J, Kwon JS, Shin YW, Lee KJ, Park S. Visual self-recognition in patients with schizophrenia. Schizophrenia Res. 2007;94(13):215-220.

3. Heinisch C, Wiens S, Grundl M, Juckel G, Brune M. Self-face recognition in schizophrenia is related to insight. Eur Arch Psychiatry Clin Neurosci. 2013;263(8):655-662.

4. Irani F, Platek SM, Panyavin IS, et al. Self-face recognition and theory of mind in patients with schizophrenia and first-degree relatives. Schizophrenia Res. 2006;88(1-3):151-160.

5. Loughland CM, Williams LM, Gordon E. Visual scanpaths to positive and negative facial emotions in an outpatient schizophrenia sample. Schizophr Res. 2002;55(1-2):159-170.

6. Delerue C, Laprevote V, Verfaillie K, Boucart M. Gaze control during face exploration in schizophrenia. Neurosci Lett. 2010;482(3):2 45-249.

7. Onitsuka T, Shenton ME, Kasai K, et al. Fusiform gyrus volume reduction and facial recognition in chronic schizophrenia. Arch Gen Psychiatry. 2003;60(4):349-355. 
8. Bortolon C, Capdevielle D, Raffard S. Face recognition in schizophrenia disorder: a comprehensive review of behavioral, neuroimaging and neurophysiological studies. Neurosci Biobehav Rev. 2015;53:79-107.

9. McCleery A, Lee J, Joshi A, Wynn JK, Hellemann GS, Green MF Meta-analysis of face processing event-related potentials in schizophrenia. Biol Psychiatry. 2015;77(2):116-126.

10. Maher S, Ekstrom T, Holt D, Ongur D, Chen Y. The core brain region for face processing in schizophrenia lacks face selectivity. Schizophrenia Bull. 2016;42(3):666-674. doi:10.1093/schbul/sbv140

11. Butler PD, Tambini A, Yovel G, et al. What's in a face? Effects of stimulus duration and inversion on face processing in schizophrenia. Schizophrenia Res. 2008;103(1-3):283-292.

12. Farah MJ, Wilson KD, Drain HM, Tanaka JR. The inverted face inversion effect in prosopagnosia: evidence for mandatory, face-specific perceptual mechanisms. Vision Res. 1995;35 (14):2089-2093.

13. Moscovitch M, Winocur G, What BM. Is special about face recognition? Nineteen experiments on a person with visual object agnosia and dyslexia but normal face recognition. $J$ Cognitive Neurosci. 1997;9(5):555-604.

14. Peelen MV, Downing PE. The neural basis of visual body perception. Nature Rev Neurosci. 2007;8(8):636-648.

15. Marquardt K, Ramezanpour H, Dicke PW, Thier P. Following eye gaze activates a patch in the posterior temporal cortex that is not part of the human "Face patch" system. eNeuro. 2017;4:2.

16. Gauthier I, Tarr MJ, Moylan J, Skudlarski P, Gore JC, Anderson AW. The fusiform "face area" is part of a network that processes faces at the individual level. J Cognitive Neurosci. 2000;12(3):495-504.

17. Ishai A, Ungerleider LG, Martin A, Haxby JV. The representation of objects in the human occipital and temporal cortex. J Cogn Neurosci. 2000;12(Suppl 2):35-51.

18. Fairhall SL, Ishai A. Effective connectivity within the distributed cortical network for face perception. Cerebral Cortex. 2007;17 (10):2400-2406.

19. Lee CU, Shenton ME, Salisbury DF, et al. Fusiform gyrus volume reduction in first-episode schizophrenia: a magnetic resonance imaging study. Arch General Psychiatry. 2002;59(9):775-781.

20. Silverstein SM, All SD, Kasi R, et al. Increased fusiform area activation in schizophrenia during processing of spatial frequency-degraded faces, as revealed by fMRI. Psychol Med. 2010;40(7):1159-1169

21. Walther S, Federspiel A, Horn H, et al. Encoding deficit during face processing within the right fusiform face area in schizophrenia. Psychiatry Res. 2009;172(3):184-191.

22. Quintana J, Wong T, Ortiz-Portillo E, Marder SR, Mazziotta JC. Right lateral fusiform gyrus dysfunction during facial information processing in schizophrenia. Biol Psychiatry. 2003;53(12):1099-1112.

23. Yovel G, Kanwisher N. The neural basis of the behavioral face-inversion effect. Current Biol. 2005;15(24):2256-2262.

24. Epstein RA, Higgins JS, Parker W, Aguirre GK, Cooperman S. Cortical correlates of face and scene inversion: a comparison. Neuropsychologia. 2006;44(7):1145-1158.

25. Steeves JK, Culham JC, Duchaine BC, et al. The fusiform face area is not sufficient for face recognition: evidence from a patient with dense prosopagnosia and no occipital face area. Neuropsychologia. 2006;44 (4):594-609.

26. Sekuler AB, Gaspar CM, Gold JM, Bennett PJ. Inversion leads to quantitative, not qualitative, changes in face processing. Current Biol. 2004;14(5):391-396.

27. Jiang X, Rosen E, Zeffiro T, Vanmeter J, Blanz V, Riesenhuber M. Evaluation of a shape-based model of human face discrimination using FMRI and behavioral techniques. Neuron. 2006;50(1):159-172.

28. James TW, Arcurio LR, Gold JM. Inversion effects in face-selective cortex with combinations of face parts. $J$ Cognitive Neurosci. 2013;25(3):455-464.
29. Matsuyoshi D, Morita T, Kochiyama T, Tanabe HC, Sadato N, Kakigi R. Dissociable cortical pathways for qualitative and quantitative mechanisms in the face inversion effect. $J$ Neurosci. 2015;35 (10):4268-4279.

30. Teunisse JP, de Gelder B. Face processing in adolescents with autistic disorder: the inversion and composite effects. Brain Cognition. 2003;52(3):285-294.

31. Hedley D, Brewer N, Young R. The effect of inversion on face recognition in adults with autism spectrum disorder. $J$ Autism Develop Disord. 2015;45(5):1368-1379.

32. Sugase-Miyamoto Y, Matsumoto N, Ohyama K, Kawano K. Face inversion decreased information about facial identity and expression in face-responsive neurons in macaque area TE. J Neurosci. 2014;34 (37):12457-12469.

33. Kovács P, Knakker B, Hermann P, Kovács G, Vidnyánszky Z. Face inversion reveals holistic processing of peripheral faces. Cortex. 2017;97:81-95.

34. Shin Y-W, Na MH, Ha TH, Kang D-H, Yoo S-Y, Kwon JS. Dysfunction in configural face processing in patients with schizophrenia. Schizophr Bull. 2008;34(3):538-543.

35. Valentine T. Upside-down faces: a review of the effect of inversion upon face recognition. Br J Psychol. 1988;79(Pt 4):471-491.

36. Richler JJ, Mack ML, Palmeri TJ, Gauthier I. Inverted faces are (eventually) processed holistically. Vision Res. 2011;51(3):333-342.

37. Kay SR, Fizzbein A, Opler LA. The positive and negative syndrome scale (PANSS) for schizophrenia. Schizophr Bull. 1987;13:261-276.

38. Tianmei S, Liang S, Yun'ai S, et al. The Chinese version of the Personal and Social Performance Scale (PSP): validity and reliability. Psychiatry Res. 2011;185(1-2):275-279.

39. Randolph C, Tierney MC, Mohr E, Chase TN. The Repeatable Battery for the Assessment of Neuropsychological Status (RBANS): preliminary clinical validity. J Clin Exp Neuropsychol. 1998;20(3):310-319.

40. Brackett MA, Salovey P. Measuring emotional intelligence with the Mayer-Salovery-Caruso Emotional Intelligence Test (MSCEIT). Psicothema. 2006;18 Suppl:34-41.

41. Kern RS, Nuechterlein KH, Green MF, et al. The MATRICS Consensus Cognitive Battery, part 2: co-norming and standardization. Am J Psychiatry. 2008;165(2):214-220.

42. Nuechterlein KH, Green MF, Kern RS, et al. The MATRICS Consensus Cognitive Battery, part 1: test selection, reliability, and validity. Am J Psychiatry. 2008;165(2):203-213.

43. Chan RC, Shi YF, Lai MK, Wang YN, Wang Y, Kring AM. The Temporal Experience of Pleasure Scale (TEPS): exploration and confirmation of factor structure in a healthy Chinese sample. PLoS One. 2012;7(4):e35352.

44. Liu WH, Wang LZ, Zhu YH, Li MH, Chan RC. Clinical utility of the Snaith-Hamilton-Pleasure scale in the Chinese settings. $B M C$ Psychiatry. 2012;12:184.

45. Xu G, Huang YX, Yan W, Luo YJ. Revision of the Chinese Facial Affective Picture System. 2011.

46. Yan C-G, Wang X-D, Zuo X-N, Zang Y-F. DPABI: data Processing \& Analysis for (Resting-State) Brain Imaging. Neuroinformatics. 2016;14(3):339-351.

47. Nichols T, Brett M, Andersson J, Wager T, Poline JB. Valid conjunction inference with the minimum statistic. NeuroImage. 2005;25 (3):653-660

48. Gold JM, Barker JD, Barr S, et al. The perception of a familiar face is no more than the sum of its parts. Psychonomic Bull Rev. 2014;21 (6):1465-1472.

49. Gold JM, Mundy PJ, Tjan BS. The perception of a face is no more than the sum of its parts. Psychol Sci. 2012;23(4):427-434.

50. Rossion B, Gauthier I. How does the brain process upright and inverted faces? Behav Cognitive Neurosci Rev. 2002;1(1):63-75.

51. Konar Y, Bennett PJ, Sekuler AB. Holistic processing is not correlated with face-identification accuracy. Psychol Sci. 2010;21 (1):38-43. 
52. Tang J, Falkmer M, Horlin C, Tan T, Vaz S, Face Recognition FT. Visual search strategies in autism spectrum disorders: amending and extending a recent review by Weigelt et al. PLoS One. 2015;10(8): e0134439.

53. Megevand P, Groppe DM, Goldfinger MS, et al. Seeing scenes: topographic visual hallucinations evoked by direct electrical stimulation of the parahippocampal place area. J Neurosci. 2014;34 (16):5399-5405.
54. Taylor SF, Chen AC, Tso IF, Liberzon I, Welsh RC. Social appraisal in chronic psychosis: role of medial frontal and occipital networks. J Psychiatric Res. 2011;45(4):526-538.

\section{Publish your work in this journal}

Neuropsychiatric Disease and Treatment is an international, peerreviewed journal of clinical therapeutics and pharmacology focusing on concise rapid reporting of clinical or pre-clinical studies on a range of neuropsychiatric and neurological disorders. This journal is indexed on PubMed Central, the 'PsycINFO' database and CAS, and is the official journal of The International Neuropsychiatric Association (INA). The manuscript management system is completely online and includes a very quick and fair peer-review system, which is all easy to use. Visit http://www.dovepress.com/testimonials.php to read real quotes from published authors. 